This item was submitted to Loughborough's Research Repository by the author.

Items in Figshare are protected by copyright, with all rights reserved, unless otherwise indicated.

\title{
Fabrication and characterisation of a novel smart suspension for micro-CMM probes
}

PLEASE CITE THE PUBLISHED VERSION

http://dx.doi.org/10.1016/j.sna.2015.04.029

PUBLISHER

(C) Elsevier

VERSION

AM (Accepted Manuscript)

\section{PUBLISHER STATEMENT}

This work is made available according to the conditions of the Creative Commons Attribution-NonCommercialNoDerivatives 4.0 International (CC BY-NC-ND 4.0) licence. Full details of this licence are available at: https://creativecommons.org/licenses/by-nc-nd/4.0/

\section{LICENCE}

CC BY-NC-ND 4.0

\section{REPOSITORY RECORD}

Alblalaihid, Khalid, Peter Kinnell, and Simon Lawes. 2015. "Fabrication and Characterisation of a Novel Smart Suspension for Micro-cmm Probes”. Loughborough University. https://hdl.handle.net/2134/21385. 


\title{
Fabrication and Characterisation of a novel smart suspension for micro-CMM probes
}

\author{
Khalid Alblalaihid $^{\mathrm{a}}$, Peter Kinnell ${ }^{\mathrm{b}} *$ and Simon Lawes ${ }^{\mathrm{a}}$ \\ a Institute for Advanced Manufacturing, the University of Nottingham, Nottingham, NG7 \\ 2RD, UK; \\ b EPSRC Centre for Innovative Manufacturing in Intelligent Automation, Wolfson School \\ of Mechanical and Manufacturing Engineering, Loughborough University, Loughborough \\ LE11 3UZ, UK; \\ * Peter Kinnell; E-Mail: P.Kinnell@lboro.ac.uk \\ Tel.: +44(0)-115-846-7680; Fax: +44(0)- 1509227146
}

\begin{abstract}
In tactile micro coordinate metrology, miniature probing systems are required to allow geometric measurements of miniature, delicate, high precision components. These probing systems typically comprise of a small stylus of only a few $\mathrm{mm}$ in length, with a spherical tip of around $100 \mu \mathrm{m}$ in diameter or less. The stylus is mounted to a flexible suspension structure which is designed to deflect during measurement, and defines the stiffness of the probing system. Stiffness is of critical importance for optimum measurement performance, and selection of the correct stiffness involves a difficult trade-off. Stiff probes are needed to overcome surface attraction forces which are significant for the small stylus tips, while flexible probes are needed for contact with delicate parts to reduce contact stress and ensure no damage is caused. To eliminate the need for compromise a novel micro tactile probing system with active stiffness control using a novel suspension structure has been designed. This paper presents the initial fabrication and the test of the suspension structure. The stiffness of the structure is assessed by measuring the modal frequencies of the suspension structure that correspond to vertical and lateral probe motion. Using this method results show it is possible to reduce the vertical and torsional frequency by $69 \%$ and $33 \%$, respectively. Using finite element analysis it is shown that this equates to vertical and lateral stiffness reductions to $12 \%$ and $46 \%$ of their initial value respectively.
\end{abstract}

Keywords: Active, Probe sensor, stiffness modulation, micro-cmm

\section{Introduction}

In recent years, micro coordinate measuring machines ( $\mu \mathrm{CMMs})$ have received increasing attention [1]. At the heart of these systems is a mechanical tactile probing system that is used to measure contact with a workpiece. In general these probing systems consist of three main parts as can be seen in Fig. 1. A long slender stylus with a spherical tip is used to make 
contact with the workpiece. The stylus transmits the contact force from the spherical stylus tip to the suspension structure. The suspension structure is designed to have relatively low stiffness such that it easily deflects as a result of contact with the workpiece. This deflection is measured by a system of displacement transducers, such as capacitive [2], optical [3], or piezo-resistive sensors [4]. While the sensor system is important, the focus of this work is the suspension structure. This structure is a critical component of the probe sensor as it determines the stiffness and sensitivity of the mechanical probe, influencing measurement performance as well as usability and durability of the probe sensor. Critically, a low stiffness probe also results in a low contact force and low stress in the workpiece, which is necessary to ensure no damage to the workpiece results from a measurement [5].

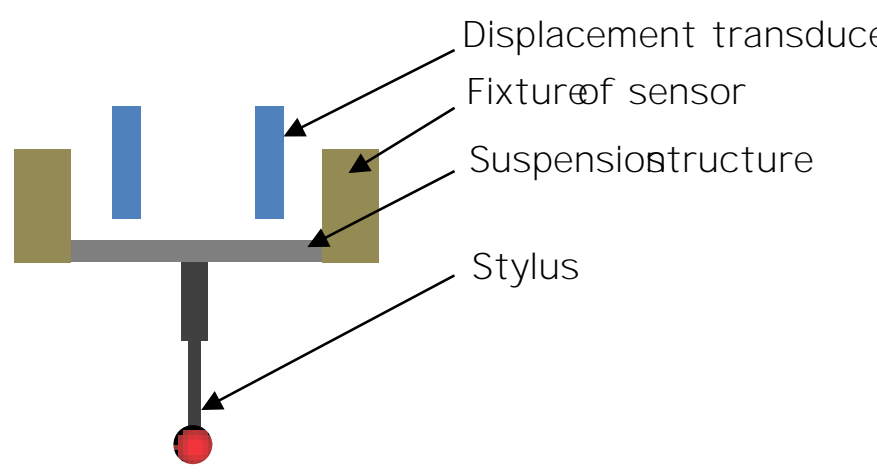

Fig. 1. Basic schematic diagram of the probing system

The problem of excessive contact stress is also set to increase as smaller probe sensors with smaller stylus tip diameters are created [6]; this is because as the stylus tip diameter is decreased the contact area for a given force also decreases, leading to an increase in contact stress. To avoid plastic-elastic deformation between the stylus tip and the workpiece two strategies are possible. The first option is to increase the sensitivity of the probe in combination with reducing probing velocity, this results in lower deflections of the probe tip during a measurement. The second option is to decrease the stiffness of the probe, leading to less contact force for a given displacement [7]. The latter option is preferable as reducing measurement speed is generally thought of as a very negative outcome; in fact efforts are normally made to increase measurement speed as much as possible.

When designing a probe sensor for low stiffness four key challenges must be addressed. The first challenge is that the probe sensor stiffness should be equal in all directions, this is termed isotropic stiffness [8]. Achieving isotropic stiffness is difficult due to the relatively long stylus that acts as a lever, causing stiffness in a direction coaxial to the stylus to be significantly higher than the stiffness in directions perpendicular to the stylus. The second challenge is that the successful manufacture of such flexible structures is not trivial, being susceptible to damage in machining and assembly [9]. The third challenge is that the low stiffness sensor should be able to overcome the attractive force experienced when the probe tip makes contact with the work piece. If the probe suspension structure is too flexible it may be damaged by surface attraction force when withdrawing $[6,9,10]$. The fourth challenge is 
that inertial loads on the suspension structure may result in significant deflection of the suspension structure without contact leading to false positive results. To mitigate this issue the accelerations applied by the CMM to move the probing system during a measurement may have to be reduced with obvious negative impact on measurement speed [11].

In this paper a novel suspension structure is presented that allows these major challenges to be addressed. It has been designed to allow active control of the probing system's stiffness during the measurement procedure. This reflects the need of the probe to be both very flexible as well as relatively stiff during different phases of a measurement. For example, before making contact with a part the stiffness should be set to be as low as possible to reduce contact stress. At this position it is also designed such that the vertical and lateral stiffness are equal to achieve isotropic stiffness. Then, after taking the measurement when the probe has made contact with the surface, stiffness may be increased so it is stiff enough to overcome surface attraction forces. Otherwise, the probe tip may become permanently stuck to the surface, or damaged as large probe deflections may be required to develop the necessary removal force.

In previous work, methods capable of modulating the stiffness of suspension structures have been evaluated [12], and piezoelectric actuators were shown to be a promising technique for stiffness control. In this work a novel smart suspension structure based on piezoelectric actuation is presented, and the working principle of the structure is explained. A prototype structure has also been manufactured and characterized, using a combination of physical testing and computer simulation to understand the factors affecting its' performance

\section{The novel smart suspension structure}

The smart suspension structure is shown in Fig. 2. It consists of a central platform suspended by three thin beams from the outer frame. The central platform is where the stylus would be fixed to create a complete probing system, in a configuration similar to that shown in Fig. 1. The three beams are the main spring elements that dictate the stiffness of the suspension structure.

To sense movement of the suspension structure during operation of the probe; the inner platform also has three cantilevered paddles which provide a suitable surface for measurement with non-contact capacitive sensors. These capacitive sensors would be located above the paddles at a stand-off distance of approximately $100 \mu \mathrm{m}$. 


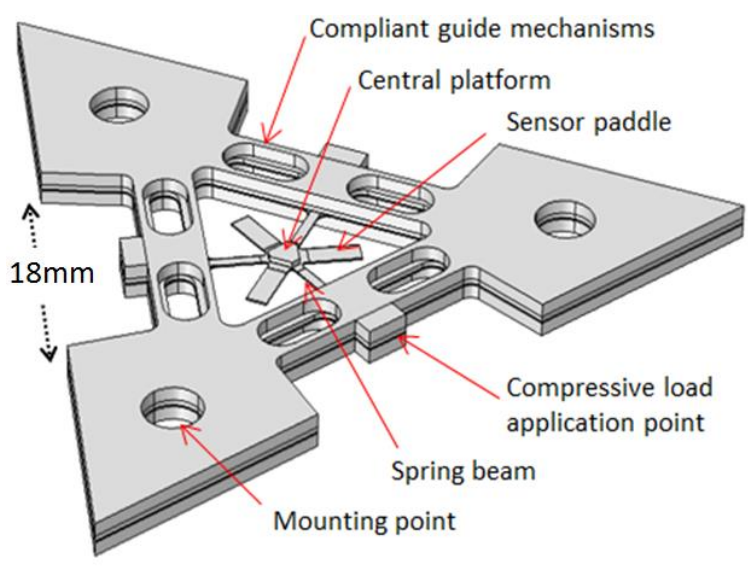

Fig. 2. Smart suspension structure, with main structural elements labelled.

The outer frame supports the spring beams and provides sturdy mounting points at the corners of the structure. These mounting points allow the structure to be assembled with the displacement sensors and mounted to a coordinate measurement machine, or in the case of the work undertaken here a test rig. The outer frame also provides well-defined load paths to transfer compressive loads to the ends of the spring beams. This is achieved by the use of the three compliant guide mechanisms that are directly connected to each spring beam. The guide mechanisms are designed to a flexible in one degree of freedom, and relatively stiff in all others. This allows them to selectively transmit uniaxial displacements along the axis of the spring beam. The compliant mechanisms are actuated by piezoelectric stacks (PICMA, P882.11) which have the ability to provide $8 \mu \mathrm{m}$ maximum displacement; the position of these actuators can be as can be seen in the top down view shown Fig. 3. The actuators are not integral to the suspension structure; instead they are positioned in intimate contact with the load application points clamped against rigid fixing locations on the test rig. The stiffness of the suspension structure is controlled by the application of a compressive force to the spring beams. As the compressive load is increased the stiffness of the beams decreases until buckling is reached. Theoretically stiffness may be reduced in this way to zero, which occurs at the buckling load for the beams. 


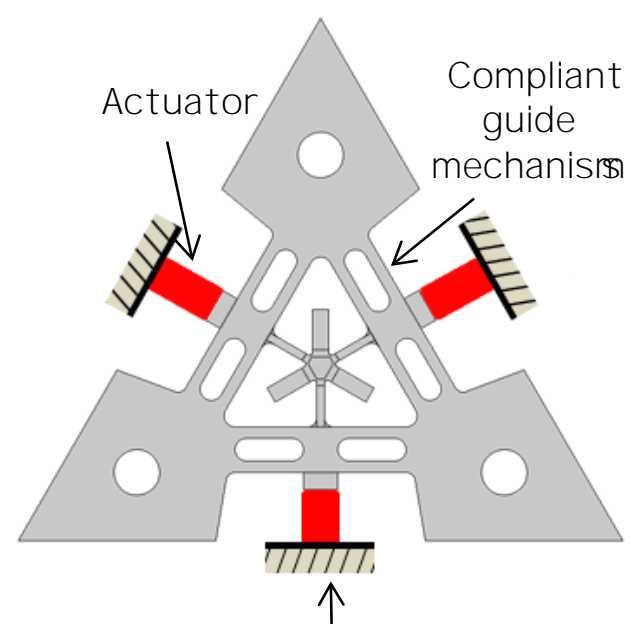

Rigid fixing points

Fig. 3. Position of the three piezoelectric actuators used to apply load to the spring beams via compliant mechanisms

\section{Manufacturing of the smart suspension structure}

To manufacture a prototype smart suspension structure, a range of manufacturing techniques were considered, and chemical etching was selected as a suitable precision manufacturing technique with details presented in preceding work[13]. The advantage of chemical etching is that it is able to remove material precisely, with no residual stress imparted to the structure. To allow the structure to be successfully manufactured using this method required it to be broken into three sub-components. These consist of a middle layer containing the spring beams, sensor paddles and central platform; and the top and bottom layers which are both identical and only contain the features associated with the outer frame.

The middle layer was manufactured from $406 \mu \mathrm{m}$ thick spring steel sheet (AISI 1095). The geometry of the structure was defined using photolithography, and was etched using a commercially available proprietary process [14]. The etching was completed in multiple stages. Two processes produce the final shape, a through-etch defines the outline of the structure, and a blind-etch defines the thickness of the spring beams. Final beam thickness was chosen to be $100 \mu \mathrm{m}$. The top and bottom layers were simpler to manufacture, requiring only one through-etch to define the outline of the structures, manufactured from $800 \mu \mathrm{m}$ thick spring steel sheet (also AISI 1095) to provide a more substantial and robust layer. Fig. 4 shows outline illustrations of the three layers. A photograph of the middle layer is given in Fig. 5 which shows the spring beam and sensor paddles with a darker surface the result of the chemical etching process used to thin these sections of the structure (the blind-etch step). 


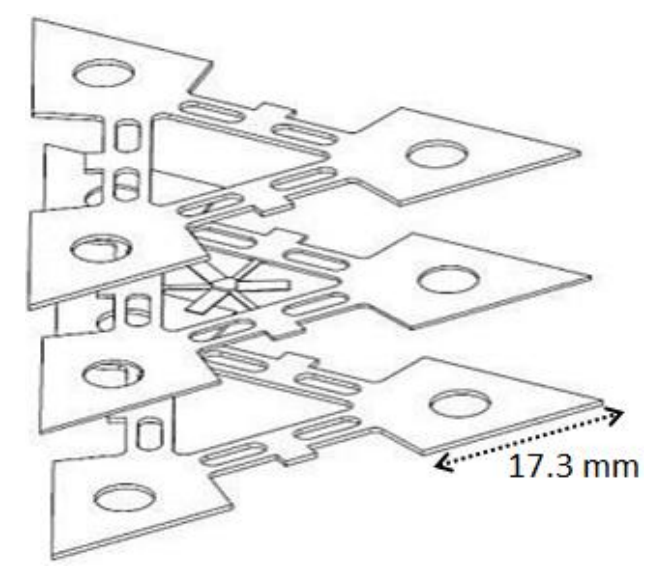

Fig. 4. Outline illustration of the three spring steel layers defined using chemical etching that form the smart suspension structure.

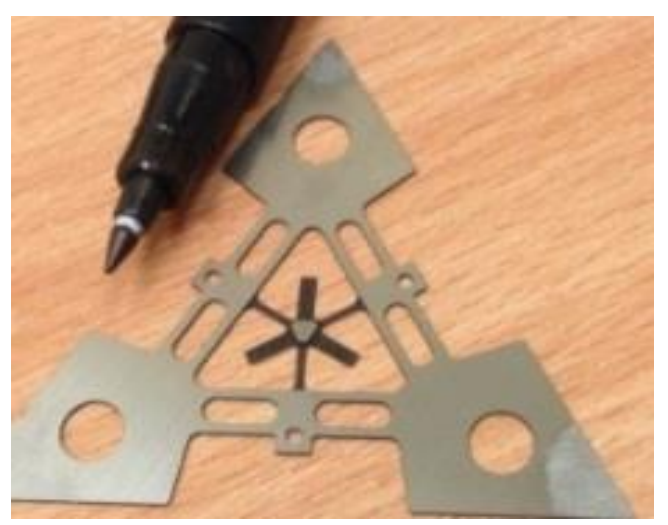

Fig. 5. Photograph of the middle layer of the smart suspension structure, the thin sections of spring beams and paddles appear to be darker in colour due to the chemical etching process.

The three layers of the smart suspension structure were then fixed together using thin layers of UHU PLUS 300 epoxy resin adhesive to create a laminar construction. This robust structure could then be mounted to a solid fixture along with the three piezoelectric actuators used to apply force to each of the three slender beams, and three capacitive sensors used to measure displacement of the sensor paddles.

\section{Suspension structure performance evaluation}

In tactile coordinate metrology the stiffness of the probing system is a critical parameter. It determines the force imparted on a part during a measurement, and is typically described by a value for both lateral and vertical stiffness. These refer to the stiffness associated with lateral and vertical displacements as illustrated in Fig. 6. These two cases are considered as they represent two extreme load cases. Due to symmetry, vertical displacement of the stylus tip results in a pure vertical load applied to the suspension structure, whereas pure lateral load results in a moment being applied to the suspension structure. For optimal performance the probe sensor design should ensure both the vertical and lateral stiffness are equal; a case 
which leads to a number of benefits in terms of minimising error sources, and simplifying the control of coordinate measurements machines when used in scanning mode $[15,16]$.

To accurately assess the vertical and lateral stiffness of a probing system requires a fully assembled probe to be tested, using an integrated precision displacement actuator and low force sensor. This would allow force displacement curves to the generated for each direction. However, in this work a complete probe was not available as only the suspension structure was manufactured. Therefore an alternative methodology was designed to assess both vertical and lateral stiffness of manufactured suspension structure. This method was based on measuring modal frequencies of the structure which are related to vertical and lateral stiffness. Structural mode shapes that are closely representative of both the vertical and lateral probing operations were selected using the results of a finite element modal analysis (details of the model are given in section 5). The selected modes can be seen displayed also in section 5, in Fig. 12. These modes were selected as they correspond to either pure vertical displacement of the central platform (which would result from vertical probing), or pure rotational displacement of the central platform (which would results from lateral probing). Therefore as the frequency of each of these modes is a function of stiffness, these frequencies may be monitored to provide an indication of how both the vertical and lateral stiffness is changing.

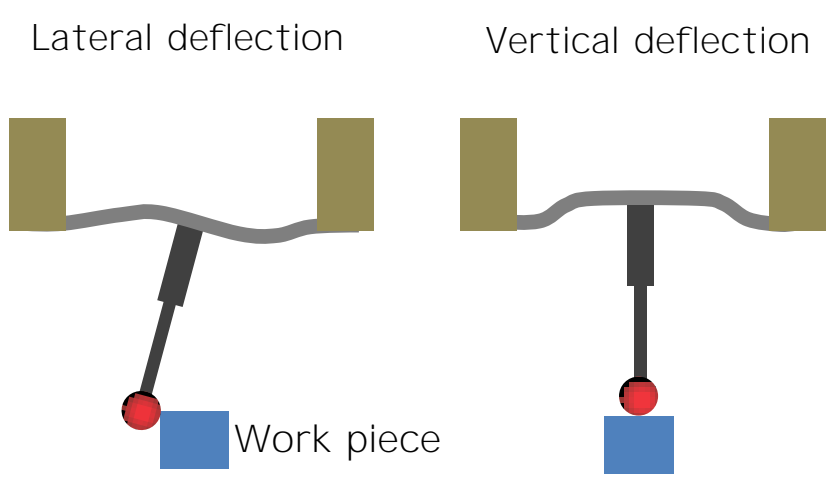

Fig. 6. Illustrating of vertical and lateral stylus tip displacements.

To test the performance of the smart suspension structure it was mounted to a fixture as shown in Fig. 7. The fixture held in place three capacitive sensors used to measure the position of the suspension structure, as well as three piezoelectric micro actuators used to apply compressive load to the structure. 


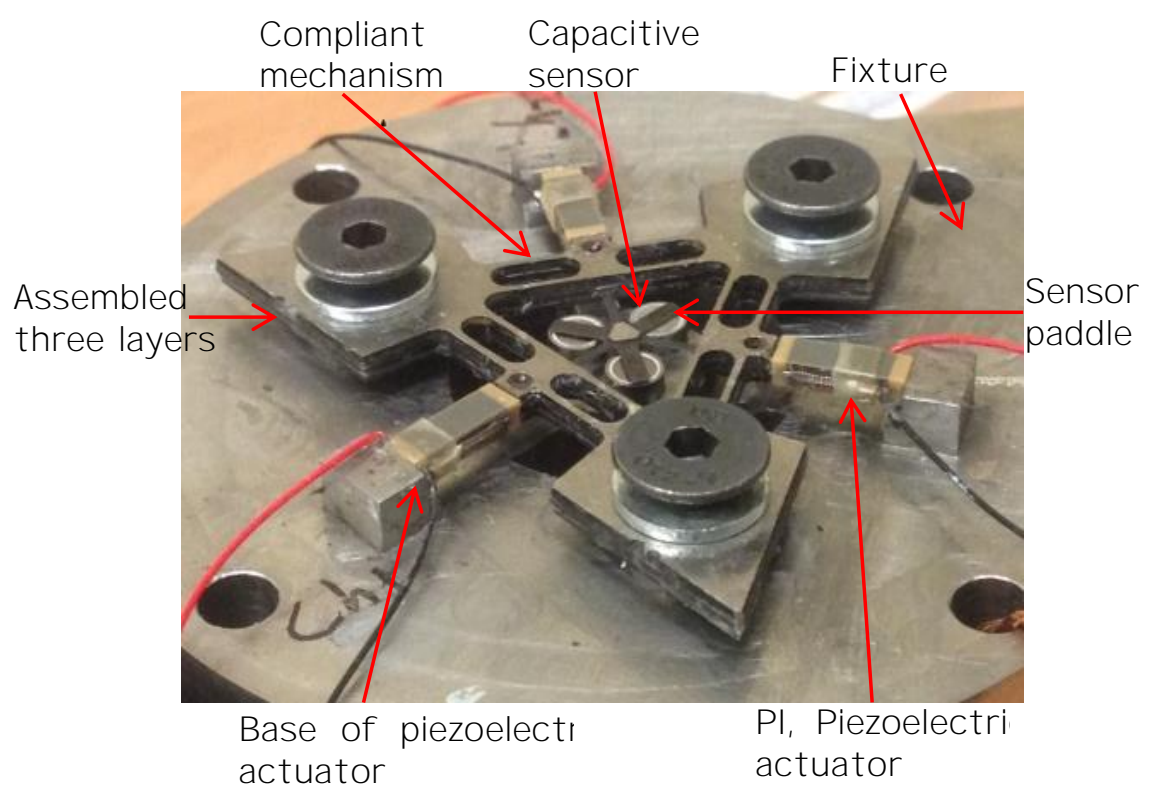

Fig. 7. The prototype of the active suspension structure

The piezoelectric actuators were used to apply a superpostion of a static load to modulate stiffness, and dynamic sinusoidal load to excite the natural frequencies of the structure. Sinusoidal excitation frequencies were applied sweeping from the $500 \mathrm{~Hz}$ to $3500 \mathrm{~Hz}$ using linear chirp signal. For a range of static loads the response of the structure to the dynamic excitation could then be determined using the three capacitive sensors. The full equipment setup is displayed in Fig. 8, this test equipment made use of a dedicated amplifier to control the voltage applied to the piezoelectric actuators (E-663, Physik Instrumente), and singal synchronisation was achieved using NI Labview and an analogue to digital converter (NI cDAQ-9172) to sample the output signals from each of the three capacitive sensors. The maximum measuring rate of the capacitive sensor was $10 \mathrm{kHz}$, which is well above the required frequency sweep range.

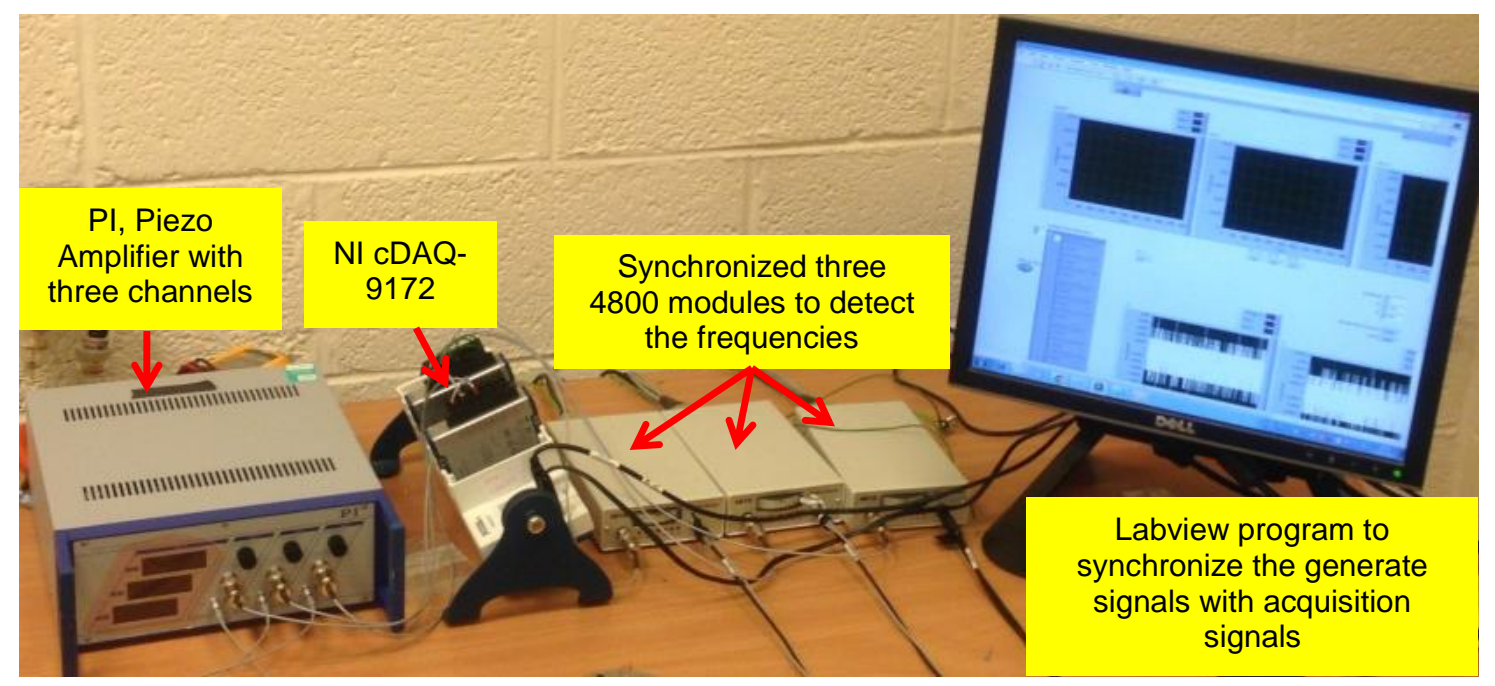

Fig. 8. Configuration and experimental setup. 
To analyse the response of the suspension structure to the chirped input signal, a Fast Fourier Transform (FFT) was performed using LabVIEW. These frequency values were then recorded with the corresponding applied static load.

For the actuators used the applied force is proportional to the applied voltage, therefore the applied voltage was used to represent applied force as an accurate calibration for this was not available. These results can be seen in the chart shown in Fig. 9, which shows two curves that initially decrease with respect to the applied voltage, and then a point of inflection is reached at approximately $60 \mathrm{~V}$ for the vertical frequency, and plateau in torsional frequency. The minimum frequency reduction, and hence stiffness reduction is therefore limited to that achieved at $60 \mathrm{~V}$ applied to the actuators.

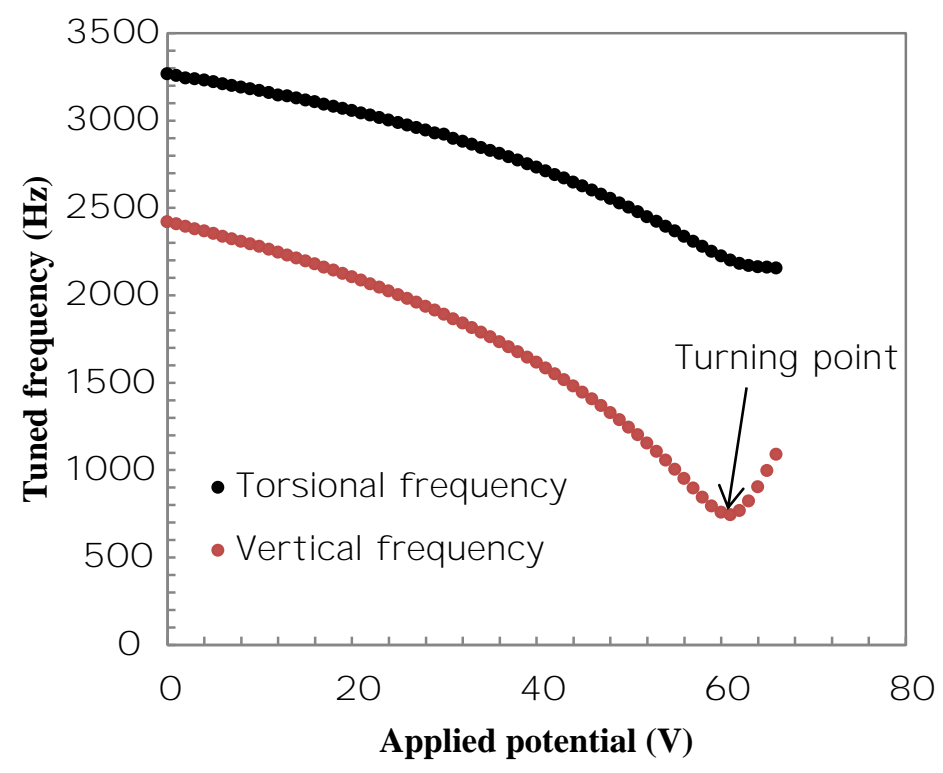

Fig. 9. A plot of the vertical and the torsional frequency of the suspension structure against the applied voltage (DC voltage), as calculated experimentally.

\section{Finite element simulation of suspension structure performance}

To understand the behavior of the structure a finite element model of exact geometry of the fabricated structure was created. All the parameters that define this geometry are presented in Table 1, and Fig. 10. The finite element model was created to match the geometry of the manufactured smart suspension structures as closely as possible and as such is able to reflect any fabrication intolerance. The geometry was measured by using a Bruker GTI contour 3D microscope. Material properties used in the model are based on values for spring steel AISI 1095 [17], which can be seen in Table 2. The finite element model was constructed so as to ensure geometric nonlinearity was accounted for. As such the effect of the axial compressive force was considered [18]. A mapped uniform mesh for each suspension beam was created to ensure an equivalent model of the properties in each beam. Quadrilateral elements were used for all suspension beams, with $154 \mu \mathrm{m}, 140 \mu \mathrm{m}$, and $25 \mu \mathrm{m}$ elements along the length, width and thickness of the beams respectively. To simplify the model only the three spring beams and the intermediate body were considered as illustrated in Fig. 10. This removed the need to model the rest of the suspension structure, which is a more 
complicated multi-layer composite structure and would add significant complexity to analysis. Also for practicality the more complex structural features, such as the parts that connect beams or the fillets, were modeled with an unstructured triangular swept mesh. An example of the output from the finite element model is given in Fig. 11, which shows results from vertical and torsional stiffness analysis of the suspension structure at zero axial compressive force where the vertical and torsional stiffness are $6648.9 \mathrm{~N} / \mathrm{m}$ and $43.9 \times 10^{-3}$ $\mathrm{Nm} / \mathrm{rad}$, respectively. The vertical stiffness has been estimated base on a load of $1 \mathrm{mN}$ in the vertical direction as chosen to be representative of typical probing forces for this type of sensor [19]. For lateral stiffness a moment of $6 \mu \mathrm{Nm}$ was chosen, based on a $1 \mathrm{mN}$ contact force applied to the end of a $6 \mathrm{~mm}$ long stylus.

In addition to the static analysis described above, the finite element model was also used to predict the modal frequencies of the suspension structure as a function of axial displacement. To simulate the deflection that was introduced by the piezoelectric actuator a series of modal analyses was carried out with a range of pre-stressed conditions. This allowed to the modal frequencies for the structure under a compressive load to be calculated. These modal frequencies were calculated to provide direct comparison to the results of the physical characterisation of the structure.

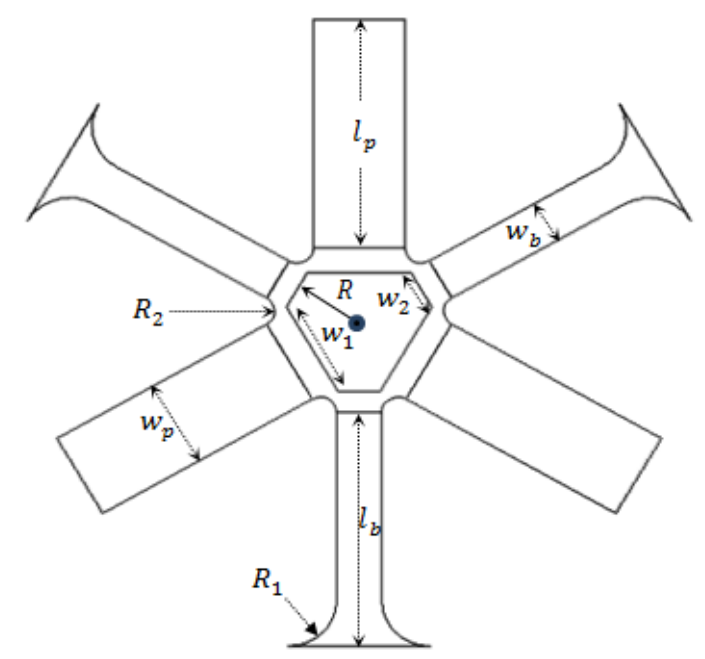

Fig. 10. The main geometric parameters of the Tri-beam design.

Table 1 Structure main parameters of the tri-beam design

\begin{tabular}{|c|c|}
\hline Item & Specifications \\
\hline Spring beams & 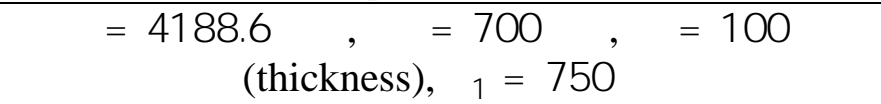 \\
\hline Central platform & 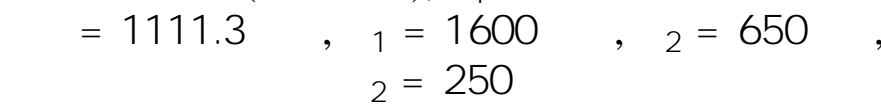 \\
\hline Sensor paddles & 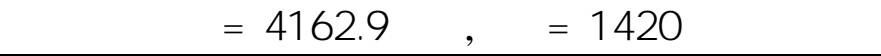 \\
\hline
\end{tabular}

Table 2 Material properties of the tri-beam design (spring steel, AISI 1095)

\begin{tabular}{cccc}
\hline Elastic & Poisson's ratio, & Density, $\rho$ & Yield strength, \\
modulus, E & $v$ & $\left(\mathrm{~kg} / \mathrm{m}^{3}\right)$ & $(\mathrm{MPa})$ \\
\hline
\end{tabular}




\begin{tabular}{cccc}
\hline$(\mathrm{GPa})$ & & & \\
\hline 210 & 0.3 & 7850 & 525 \\
\hline
\end{tabular}

a.

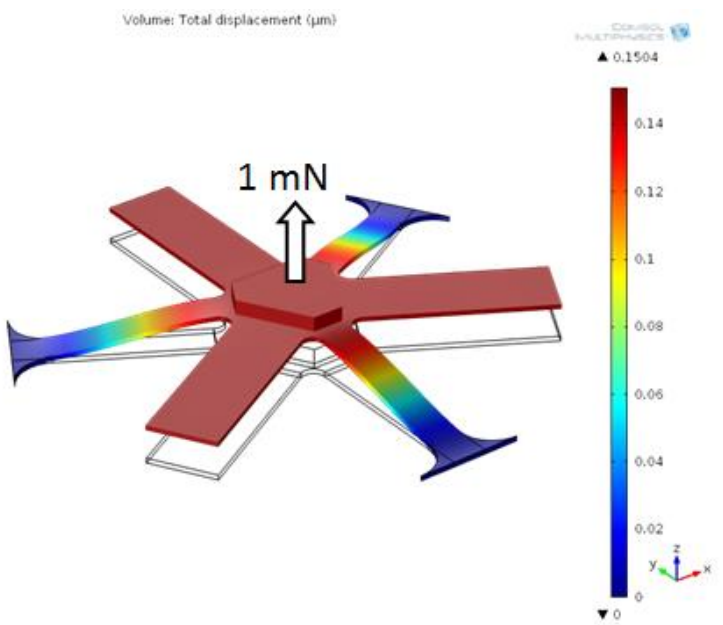

b.

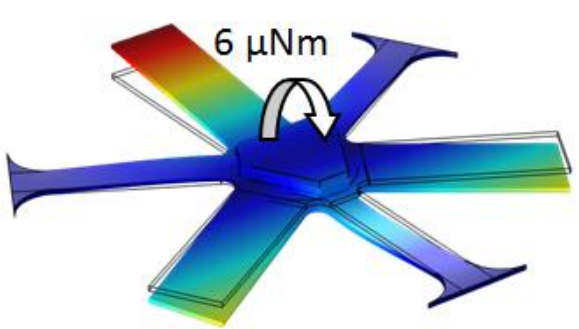

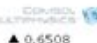

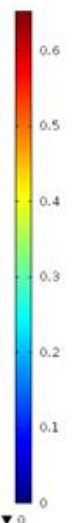

Fig. 11. Shows results for zero applied compressive load: (a) Vertical displacement resulting from an applied vertical load of $1 \mathrm{mN}$ at the centre of the structure, (b) torsional stiffness analysis of the suspension structure resulting from a $6 \mu \mathrm{Nm}$ at the centre of the structure.

The first two mode shapes were estimated numerically using COMSOL to identify the expected modal frequencies, and their associated mode shapes, these are shown in Fig. 12; which shows that the suspension structure vibrates vertically at $2402.6 \mathrm{~Hz}$, with the second modes representing a torsional motion with a frequency of $3061.5 \mathrm{~Hz}$.

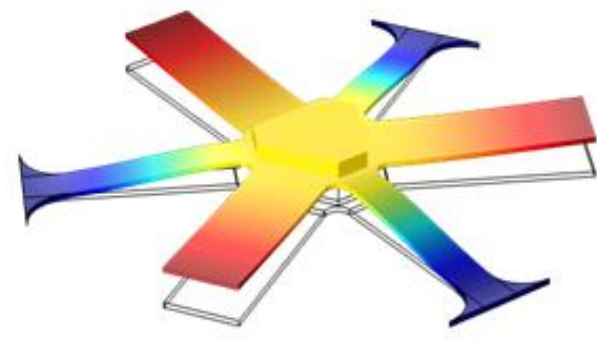

a. Frequency $=2402.6 \mathrm{~Hz}$

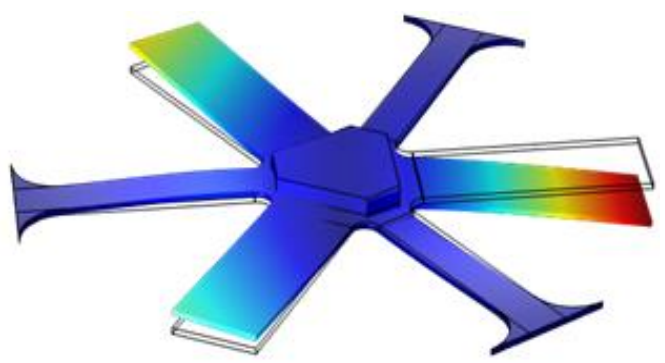

b. Frequency $=3061.5 \mathrm{~Hz}$

Fig. 12. Shows mode shapes for zero applied compressive load: (a.) the first mode, where the suspension structure vibrates vertically, and (b.) the second mode where the centre of the structure has a torsional motion.

This analysis was run over a range of applied axial displacements; results can be seen in the chart in Fig. 14. The solid lines on the chart represent the model of the manufactured suspension structure, by inspection it can be seen that the shape of the curves for the torsional and vertical modes are similar to those collected experimentally (see Fig. 9). The simulation was able to predict with good agreement the behaviour of the structure and in particular the limited frequency reduction beyond a displacement limit as was seen for both modes in the physical device. A potential cause of this was thought to be non-symmetrical beam geometry as a result of the chemical etching process, which required the suspension structure beams to 
be manufactured with single-sided blind etching only as illustrated in Fig. 13. To understand the impact of this on the performance of the structure, a slightly modified version of the finite element model was created. This second model was designed to be overall the same dimensions as the first, however the beams were modelled as completely symmetrical as would be produced from a double sided blind etch (see Fig. 13). The results collected from this model are also shown on the chart in Fig. 14 as the broken lines. For the symmetrical model it can be seen that the structure is able to reduce modal frequencies down to zero.

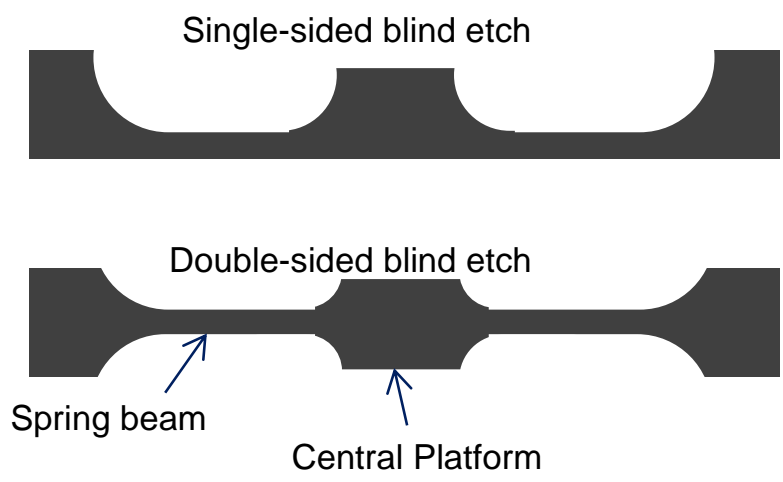

Fig. 13. Schematic diagram of the cross-section of a beam etched using two different strategies to demonstrate non-symmetrical nature of a beam manufactured with a single-sided etch as compared to a double-sided etch.

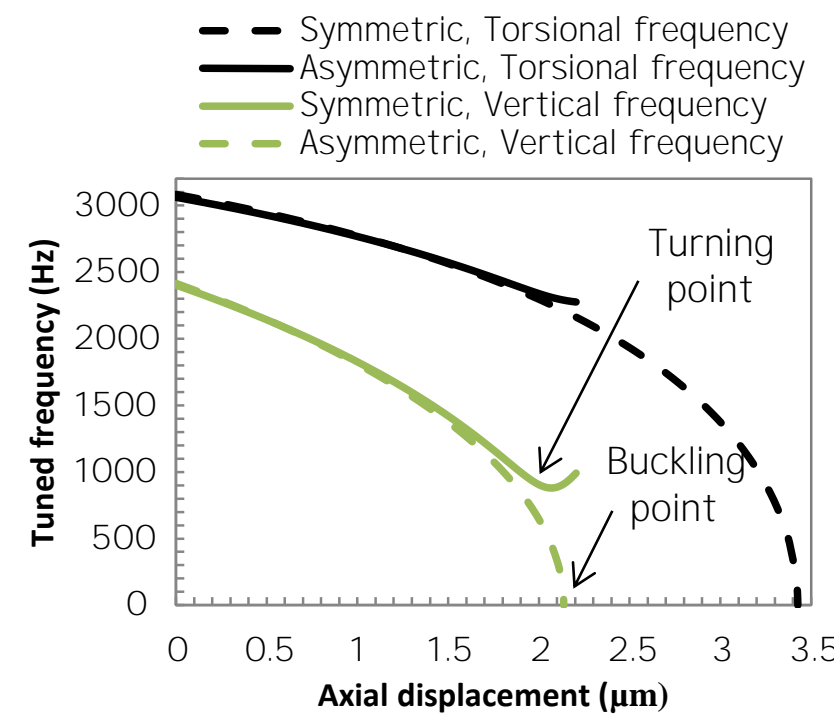

Fig. 14. Graph of modal frequency against axial displacement of the suspension structure, as calculated for symmetric and asymmetric beam in FAE analysis (COMSOL).

\section{Analysis of results and discussion}

Considering the experimental test results it is clear that the strucure is able to successfully modulate stiffness. The results in Fig. 9 show that for both the torsional and vertical modes of the suspension structure it was possible to reduce frequency by applying a control voltage to 
the actuators. As the applied voltage was proportional to applied load, these results show that as load is applied to the structure the frequency and therefore its' stiffness is reduced. The reduction in frequency was limited though as a turning point was reached at a load corresponding to a frequency of approximately $743 \mathrm{~Hz}$ for the vertical mode. This response was also seen in the finite element modal analysis for asymmetric beams as can be seen in Fig. 14. To allow direct comparison of the model data with the experimental data, the aplied voltage used in the experimental setup and the applied displacement used in the finite element model were both normalised to one scale. This can be done if we assume the voltage applied scales linearly with applied load and displacement. The scaling was done by normalinsing both sets of data to a value of one at the observed minimum vertical frequency. The results from this can be seen in Fig. 15, where it can be seen that the finite element model is in reasonable agreement with the experimental data. The exact source of the observed errors was not determined, however the scale of the errors was small with the maximum the differnece between observed and predicted frequencies being only $6 \%$. This small error may be due to the simlifications made to the finited element model which is only considereing the spring beams and intermediate body. Further detail of the results is given in Table 3, which presents a comparison of the measured frequencies, and those predicated by the finite element model for the suspension structure; by considering the initial frequency and the lowest possible frequency as a result of applied displacement or voltage.

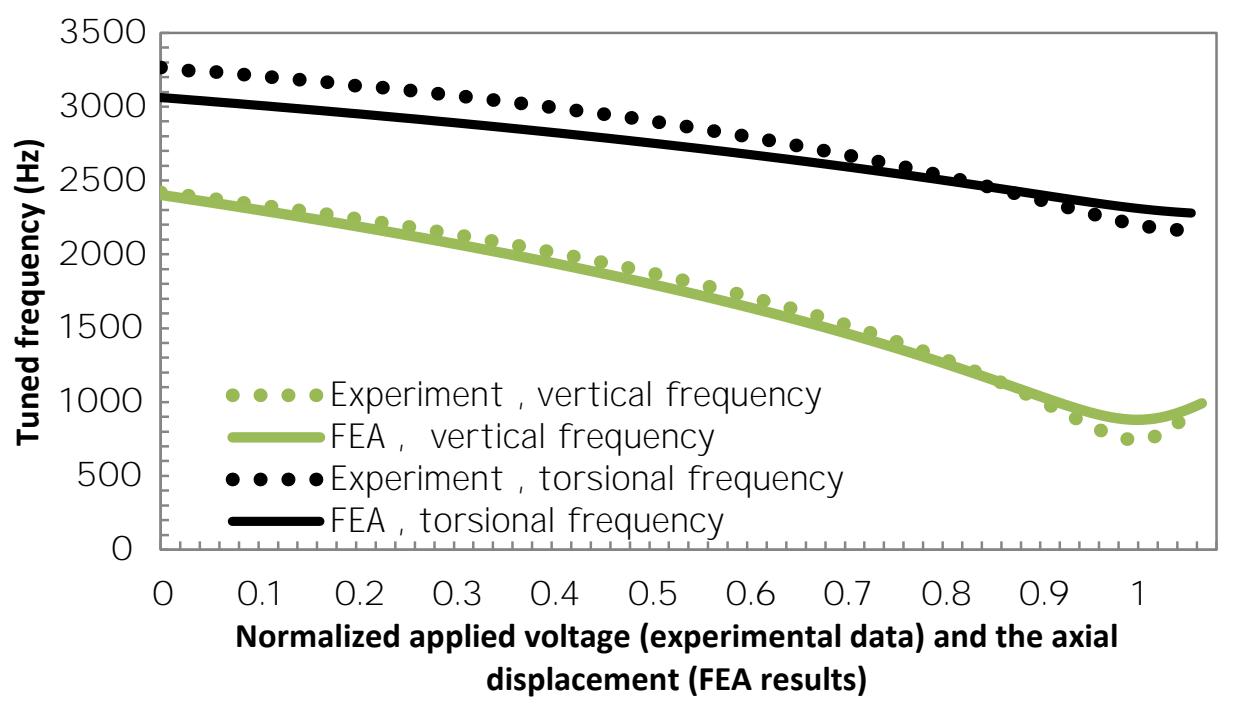

Fig. 15. Graph of modal frequency of the suspension structure against the normalized scale at the observed minimum frequency for the vertical direction, as calculated for asymmetric beam in experimental result and FAE analysis.

Comparison of data clearly also shows that the maximum level of frequency reduction possible is limited by the structral asymmetry that resulted from the prototype manufacture. To explain this consider any buckling beam system. If the system is complete symmetrical the beam will be able to have compressive load applied until the buckling point is reach, at which point it will be come unstable and the stiffness will reduce to zero. If asymmetry is 
introduced to the system, either in the form of additional off-centre loading, or as in this case non-symentrical geometry, then the strucutre will be natrually subjected to bending stresses. These bending stresses predispose the system to bend before it buckles, and when bending occurs there is a small relief in the applied compressive load. This is a highly non-linear effect, as such it is not felt until a significant level of compressive load has been applied. In the case of the smart suspension structure it is visible at the turning point as the frequency stops decreasing as a result of applied load, and then starts to increase as the induced bending negates the compressive loads.

To quantify the observed frequency change in terms of stiffness, the finite element model was used to predict the stiffness change made possibly once the turnign point was reached. This was done assuming the suspension structure would be used as part of a probing system with a $6 \mathrm{~mm}$ stylus length. From the model the minimum vertical stiffness was found to be $788 \mathrm{~N} / \mathrm{m}$, approximately $12 \%$ of the initial stiffness of $6648 \mathrm{~N} / \mathrm{m}$. While, for the lateral stiffness a minimum of $562 \mathrm{~N} / \mathrm{m}$ was found which is $46 \%$ of the initial stiffness of 1219 $\mathrm{N} / \mathrm{m}$. As a key consideration for probe system design is to have isotropic stiffness the ratio of the vertical to lateral stiffnesses is considered. For the initial stiffness of the strucutre this ratio would be 5.4, while for the minimum stiffness condition this ratio is reduced to 1.4 which is close to the isotropic stiffness.

Table 3 Comparison of the measured and the finite element predicted frequencies for the suspension structure.

\begin{tabular}{|c|c|c|c|c|c|c|c|c|}
\hline \multirow{3}{*}{$\begin{array}{l}\text { Type of } \\
\text { frequency }\end{array}$} & \multirow{2}{*}{\multicolumn{3}{|c|}{ Experiment }} & \multicolumn{5}{|c|}{ COMSOL (FEA) } \\
\hline & & & & \multicolumn{2}{|c|}{ Asymmetric } & \multicolumn{3}{|c|}{ Symmetric } \\
\hline & 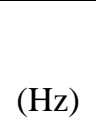 & $\begin{array}{r}{ }^{-} \text {ө日 } \\
(\mathrm{Hz})\end{array}$ & $\begin{array}{c}\text { Reducation } \\
(\%)\end{array}$ & $\begin{array}{l}{ }^{\underline{u}} \delta \\
(\mathrm{Hz})\end{array}$ & $\begin{array}{r}{ }^{u} \text { ө } \mathrm{GH} \\
(\mathrm{Hz})\end{array}$ & $\begin{array}{c}\text { Reducation } \\
(\%)\end{array}$ & $\begin{array}{l}{ }_{\mathcal{u}} \\
(\mathrm{Hz})\end{array}$ & 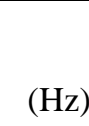 \\
\hline Vertical & 2420 & 743 & 69 & 2403 & 880 & 63 & 2415 & 0 \\
\hline Torional & 3265 & 2200 & 33 & 3062 & 2311 & 25 & 3083 & 0 \\
\hline
\end{tabular}

\section{Conclusion and future work}

In this work the first ever example of a variable stiffness suspension structure for micro tactile probing systems has been designed, fabricated, and tested. This variable stiffness suspension structure overcomes challenges in tactile metrology that are not addressed by any other commercial systems. Using this technique allows the stiffness of the suspension structure to be modulated during probing, avoiding many practical issues related to the stiffness of the probe being either too high or too low at different phases of a measurement. An analysis of the modal frequencies of the structure was undertaken to demonstrate that the stiffness modulation method was able to reduce the frequency of the vertical mode by 
approximately $70 \%$, while the torsional mode was decreased by approximately $33 \%$. This level of reduction was shown to be in close agreement with a finite element simulation of the structure. Using the finite element model it was shown that vertical and lateral stiffness could be reduced to $12 \%$ and $46 \%$ of their initial value respectively. Moreover the ratio between these two stiffness values was reduced from 5.4 to only 1.4 .

While the presented reduction in stiffness would represent a significant benefit, it has also been shown that the factors limiting the further reduction of stiffness are well understood and can be effectively modeled using the finite element method. It is therefore anticipated that further design and manufacturing process optimisation will result in a significant capability to provide even more stiffness reduction, and achieved perfect isotropic stiffness.

To fully demonstrate the feasibility of this technique, further work is ongoing in the embodiment of an optimized suspension structure that provides higher stiffness reduction. Moreover, integrating a stylus with a suspension structure to allow a true measure of the actual probe stiffness will be assessed in future work. Once this is realized a full assessment of the factors affecting measurement stability, such as long and short term off-set drift, sensitivity drift, and thermal coefficients for this type of probing system must be established.

\section{Reference}

[1] A. Weckenmann, G. Peggs, and J. Hoffmann, "Probing systems for dimensional micro- and nano-metrology," Measurement Science and Technology, vol. 17, p. 504, 2006.

[2] T. Liebrich and W. Knapp, "Improvements and experimental validation of a 3Dprobing system for micro-components," CIRP Annals - Manufacturing Technology, vol. 61, pp. 475-478, 2012.

[3] K.-C. Fan, F. Cheng, W. Wang, Y. Chen, and J.-Y. Lin, "A scanning contact probe for a micro-coordinate measuring machine (CMM)," Measurement Science and Technology, vol. 21, p. 054002, 2010.

[4] E. J. C. Bos, "Aspects of tactile probing on the micro scale," Precision Engineering, vol. 35, pp. 228-240, 2011.

[5] F. Meli and A. Küng, "AFM investigation on surface damage caused by mechanical probing with small ruby spheres," Measurement Science and Technology, vol. 18, p. 496, 2007.

[6] S.-M. Kao and D.-Y. Sheu, "Developing a novel tri-switch tactile probing structure and its measurement characteristics on micro-CMM," Measurement, vol. 46, pp. 3019-3025, 2013.

[7] E. J. C. Bos, F. L. M. Delbressine, and H. Haitjema, "High-accuracy CMM metrology for micro products," in IMEKO conference, 2004.

[8] F. G. Balzer, T. Hausotte, N. Dorozhovets, E. Manske, and G. Jäger, "Tactile 3D microprobe system with exchangeable styli," Measurement Science and Technology, vol. 22, p. 094018, 2011.

[9] E. J. C. Bos, "Tactile 3D probing system for measuring MEMS with nanometer uncertainty.," Phd, Eindhoven University of Technology, Netherlands, 2008. 
[10] J. D. Claverley and R. K. Leach, "Development of a three-dimensional vibrating tactile probe for miniature CMMs," Precision Engineering, vol. 37, pp. 491-499, 2013.

[11] A. Weckenmann, T. Estler, G. Peggs, and D. McMurtry, "Probing Systems in Dimensional Metrology," CIRP Annals - Manufacturing Technology, vol. 53, pp. 657684, 2004.

[12] K. Alblalaihid, P. Kinnell, and S. Ratchev, "An evaluation of stiffness modulating techniques for a micro-CMM tactile probe," in 14th euspen International Conference, Dubrovnik, Croatia, 2014.

[13] K. Alblalaihid, T. Kirk, S. Lawes, and P. Kinnell, "Fabrication of a smart suspension structure of micro tactile probing," in EUROSENSORS, Italy, Brescia, 2014.

[14] PrecisionMicro. (2014, September). Available: www.precisionmicro.com

[15] V. Nesterov and U. Brand, "Modelling and investigation of the silicon twin design 3D micro probe," Journal of Micromechanics and Microengineering, vol. 15, p. 514, 2005.

[16] A. Tibrewala, N. Hofmann, A. Phataralaoha, G. Jäger, and S. Büttgenbach, "Development of 3D Force Sensors for Nanopositioning and Nanomeasuring Machine," Sensors, vol. 9, pp. 3228-3239, 2009.

[17] S. Dhar, R. Sethuraman, and P. M. Dixit, "A continuum damage mechanics model for void growth and micro crack initiation," Engineering Fracture Mechanics, vol. 53, pp. 917-928, 1996.

[18] COMSOL, "Multiphysics Reference Guide for COMSOL 4.3a," ed, 2013.

[19] J. B. Tan and J. N. Cui, "Ultraprecision 3D probing system based on spherical capacitive plate," Sensors and Actuators A: Physical, vol. 159, pp. 1-6, 2010. 


\section{Table legends}

Table 1 Structure main parameters of the tri-beam design

Table 2 Material properties of the tri-beam design (spring steel, AISI 1095)

Table 3 Comparison of the measured and the finite element predicted frequencies for the suspension structure 


\section{Figure legends}

Fig. 1. Basic schematic diagram of the probing system.

Fig. 2. Smart suspension structure, with main structural elements labelled.

Fig. 3. Position of the three piezoelectric actuators used to apply load to the spring beams via compliant mechanisms.

Fig. 4. Outline illustration of the three spring steel layers defined using chemical etching that form the smart suspension structure.

Fig. 5. Photograph of the middle layer of the smart suspension structure, the thin sections of spring beams and paddles appear to be darker in colour due to the chemical etching process.

Fig. 6. Illustrating of vertical and lateral stylus tip displacements.

Fig. 7. The prototype of the active suspension structure.

Fig. 8. Configuration and experimental setup.

Fig. 9. A plot of the vertical and the torsional frequency of the suspension structure against the applied voltage (Dc voltage), as calculated experimentally.

Fig. 10. The main geometric parameters of the Tri-beam design.

Fig. 11. Shows results for zero applied compressive load: (a) Vertical displacement resulting from an applied vertical load of $1 \mathrm{mN}$ at the centre of the structure, (b) torsional stiffness analysis of the suspension structure resulting from a $6 \mu \mathrm{Nm}$ at the centre of the structure.

Fig. 12. Shows mode shapes for zero applied compressive load: (a.) the first mode, where the suspension structure vibrates vertically, and (b.) the second mode where the centre of the structure has a torsional motion.

Fig. 13. Schematic diagram of the cross-section of a beam etched using two different strategies to demonstrate non-symmetrical nature of a beam manufactured with a single-sided etch as compared to a double-sided etch.

Fig. 14. Graph of modal frequency against axial displacement of the suspension structure, as calculated for symmetric and asymmetric beam in FAE analysis (COMSOL).

Fig. 15. Graph of modal frequency of the suspension structure against the normalized scale at the observed minimum frequency for the vertical direction, as calculated for asymmetric beam in experimental result and FAE analysis. 